\section{Detrimental effects of intracerebral haemorrhage on patients with CADASIL harbouring NOTCH3 R544C mutation}

\section{INTRODUCTION}

Cerebral autosomal dominant arteriopathy with subcortical infarct and leukoencephalopathy (CADASIL) caused by mutations of the 33-exon NOTCH3 gene is the most common single gene disorder of stroke. ${ }^{1}$ To date, more than 200 different NOTCH3 mutations have been reported worldwide, while R544C in exon 11 was the most prevalent hot spot mutation in East Asia and accounted for more than $70 \%$ of the patients in their CADASIL cohorts. $^{2} 3$ Patients with CADASIL may suffer from ischaemic stroke (IS) and intracerebral haemorrhage (ICH), especially among Asians. Therefore, we aimed to compare the clinical features of acute ICH vs IS events in patients with CADASIL and focused on the effects of ICH on stroke recurrence and long-term outcome.

\section{METHODS}

The patients enrolled in this study were prospectively recruited from a multicentre stroke registry established since 2006 with at least 12 hospitals in Taiwan. We screened R544C mutation from participants who provided their blood sample and those whose harbouring R544C mutation were then included in the present study. Preadmission, inpatient and discharge data were collected by trained personnel. The responsible neurologist on each study site will examine the medical record or interview the patients to acquire follow-up information. All patients received at least one neuroimaging examination to document the locations of acute stroke. Abnormal white matter hyperintensity
(WMH) lesion was scored by the Fazekas scale. The number and distribution of cerebral microbleeds (CMBs) were also documented.

Clinical and neuroimaging features were compared using the t-test, Mann-Whitney $U$ test and $\chi^{2}$ test when appropriate. Kaplan-Meier plots of recurrent stroke survival were computed for the ICH-onset versus IS-onset groups and compared with the log-rank test. A Cox proportional hazards regression model was then used to estimate the HR and 95\% CI for recurrent stroke.

\section{RESULTS}

From the registry consisting of nearly 2000 stroke patients, 71 (mean age at first stroke, 58.39 .6 years; $63 \%$ male) were screened positive for R544C mutation, and 103 stroke events of them were documented ( 37 ICH and 64 IS events). The initial stroke severity, functional outcome at discharge, and mortality were significantly higher in the ICH events. The mean $\mathrm{ICH}$ volume was $10.9 \mathrm{ml}$, and the most common locations of $\mathrm{ICH}$ were thalamus $(51 \%)$ and basal ganglia (27\%). Among the IS events, subcortical white matter was the predominant stroke location (41\%), and small vessel occlusion type accounted for more than half $(59 \%)$ of stroke cases.

Of 67 patients with a median follow-up time of 66 months (IQR, 39-106), 21 (31\%) patients were classified as ICH-onset, 29 patients had recurrent strokes and 10 patients died. Patients with ICH-onset were more likely to have high family burden of stroke (at least two first-degree relatives with stroke), slightly higher WMH score and higher total CMBs count (table 1). The annual stroke recurrence rate was 8.1 per 100 person-years (95\% CI 5.6 to 11.3) in all patients and was higher in the ICH-onset than IS-onset group (10.9 vs 7.0 per 100 person-years, log-rank $=0.03 ;$ online supplementary figure 1). The recurrent type of stroke may be either haemorrhagic or ischaemic or even both. After adjustment for age at first stroke, gender and hypertension, patients with ICH-onset still carried a higher risk of recurrent stroke (HR 2.17, $95 \%$ CI 1.01 to $4.64, p=0.05)$. Overall, $28(42 \%)$ patients suffered from at least one ICH events. Patients with ever ICH had significantly higher mortality rate, family burden of stroke, WMH lesion, and more $\mathrm{CMBs}$ count than those with IS-only (online supplementary table 1). 
Table 1 Clinical and neuroimaging features of patients with CADASIL with ICH-onset vs ISonset

\begin{tabular}{llll}
\hline & ICH-onset $(\mathbf{n}=\mathbf{2 1})$ & IS-onset $(\mathbf{n}=\mathbf{4 6})$ & $\boldsymbol{P}$ values \\
\hline Age (at first stroke) & $58.3 \pm 9.1$ & $57.8 \pm 9.9$ & 0.85 \\
\hline Male & $14(66.7)$ & $28(60.9)$ & 0.65 \\
\hline Annual recurrent rate & $10.9 / 100$ person-years & $7.0 / 100$ person-years & $0.03^{*}$ \\
\hline Recurrence stroke & $12(57.1)$ & $17(37.0)$ & 0.12 \\
\hline Only IS & 5 & 10 & 0.66 \\
\hline Only ICH & 6 & 6 & \\
\hline Both ICH and IS & 1 & 1 & \\
\hline Mortality & $6(28.6)$ & $4(8.7)$ & 0.06 \\
\hline Family history of stroke & $11(52.4)$ & $19(41.3)$ & 0.40 \\
\hline High family burden of stroke & $7(33.3)$ & $5(10.9)$ & 0.03 \\
\hline Hypertension & $19(90.5)$ & $36(78.3)$ & 0.23 \\
\hline Diabetes mellitus & $6(28.6)$ & $13(28.3)$ & 0.98 \\
\hline Hyperlipidaemia & $12(57.1)$ & $22(47.8)$ & 0.48 \\
\hline Smoking & $7(33.3)$ & $20(43.5)$ & 0.43 \\
\hline WMH scoret & $2.9 \pm 0.3$ & $2.6 \pm 0.7$ & 0.16 \\
\hline Anterior temporal WMH & $1(8.3)$ & $5(11.4)$ & 0.99 \\
\hline External capsule WMH & $10(83.3)$ & $30(68.2)$ & 0.48 \\
\hline Numbers of lacunes & $5.2 \pm 4.7$ & $6.3 \pm 5.9$ & 0.58 \\
\hline Number of CMBs & $27.4 \pm 23.8$ & $12.5 \pm 17.5$ & 0.05 \\
\hline Location of CMBs: none & 0 & 8 & 0.14 \\
\hline Pure deep & 1 & 6 & \\
\hline Pure lobar & 0 & 1 & \\
\hline Mixed & 8 & 13 & \\
\hline Data we & & & \\
\hline
\end{tabular}

Data were presented by count number (percentage) or mean SD.

${ }^{*}$ Compared with log-rank test.

tOnly 56 patients (12 in ICH, 44 in IS group) had available MRI.

‡Only 37 patients (9 in ICH, 28 in IS group) had available CMBs imaging.

CADASIL, cerebral autosomal-dominant arteriopathy with subcortical infarct and leukoencephalopathy; CMBs,

cerebral microbleeds; ICH, intracerebral haemorrhage; IS, ischaemic stroke; WMH, white matter hyperintensity.

\section{DISCUSSION}

The present study demonstrated that up to $40 \%$ patients with CADASIL harbouring R544C mutation could develop ICH during the follow-up course. The impacts of ICH on functional outcome were huge and included increased risk of stroke recurrence and mortality. The risk factors for ICH identified in these patients were strong family history of stroke and severe white matter changes on neuroimaging.

There were several novel findings from our study. First, we described 37 ICH events on 28 patients of CADASIL, which is by far the largest case series to date. Apart from scatter case reports, one recent Korea study reported $22 \mathrm{ICH}$ events out of 16 patients and another Taiwan study presented 18 cases of clinical or neuroimaging history of $\mathrm{ICH}^{3}{ }^{34}$ Interestingly, all these studies came from area where R544C mutation was prevalent. Second, a substantial high proportion (up to $30 \%$ ) of patients had ICH as their initial presentation, and they were prone to have recurrent stroke and higher risk of mortality. This finding also implied that clinicians should consider the possibility of CADASIL even in patients with $\mathrm{ICH}$, especially when they had strong family history of stroke, severe WMH lesion or high burden of CMBs. In addition, the prevalence of hypertension was high in our group, and the most common locations of ICH were also compatible with typical hypertensive haemorrhage. Whether a specific R544C phenotype of CADASIL vasculopathy, or a superimposed hypertensive arteriopathy, was responsible for the occurrence of ICH required further investigation. ${ }^{45}$

There were limitations of our study. First, patients recruited from the stroke registry were only screened for R544C mutation because it accounted for more than $70 \%$ of $\mathrm{NOTCH} 3$ mutation in Taiwan. Consequently, our findings may not be applicable to other mutation sites of $\mathrm{NOTCH} 3$ or other populations. Second, our study included patients with stroke only. Patients with CADASIL without stroke but with other manifestations were not included, thus we could not identify crucial background factors related to the occurrence of stroke.

In conclusion, the present study found ICH events in a high proportion of patients with CADASIL with NOTCH3 mutation R544C. Subjects with ICH as their first stroke event had significantly higher risk of recurrent stroke and mortality. Further study to determine the appropriate management of patients with CADASIL is essential to both preventing ischaemic and avoiding haemorrhagic events.

Chih-Hao Chen, ${ }^{1,2}$ Sung-Chun Tang, ${ }^{1}$ Yu-Wen Cheng, ${ }_{1}^{1}$ Hsin-Hsi Tsai, ${ }^{1}$ Nai-Fang Chi, ${ }^{3}$ Pi-Shan Sung ${ }^{4}{ }^{4}$ Hsu-Ling Yeh, ${ }^{5}$ Li-Ming Lien, ${ }^{5}$ Huey-Juan Lin, ${ }^{6}$ Ming-Jen Lee, ${ }^{1}$ Chaur-Jong $\mathrm{Hu}^{3}{ }^{3}$ Hung-Yi Chiou, ${ }^{7}$ Jiann-Shing Jeng ${ }^{1}$

${ }^{1}$ Stroke Center and Department of Neurology, National Taiwan University Hospital, Taipei, Taiwan

${ }^{2}$ Graduate Institute of Epidemiology and Preventive Medicine, College of Public Health, National Taiwan University, Taipei, Taiwan

${ }^{3}$ Department of Neurology, Taipei Medical University Hospital and Shuang Ho Hospital, Taipei, Taiwan ${ }^{4}$ Stroke Center and Department of Neurology, National Cheng Kung University Hospital, Tainan, Taiwan ${ }^{5}$ Department of Neurology, Shin Kong Wu Ho-Su Memorial Hospital, Taipei, Taiwan

${ }^{6}$ Department of Neurology, Chi-Mei Medical Center, Tainan, Taiwan

${ }^{7}$ School of Public Health, College of Public Health, Taipei Medical University, Taipei, Taiwan

Correspondence to Dr Sung-Chun Tang, Department of Neurology, National Taiwan University Hospital, Taipei 10055, Taiwan; tangneuro@gmail.com

Contributors C-HC and S-CT planned the study. All authors contributed to data acquisition. C-HC, Y-WC and H-HT analysed the data. C-HC wrote the first draft. S-CT and J-SJ revised the draft paper. All authors reviewed the manuscript and approved the final version.

Funding The authors have not declared a specific grant for this research from any funding agency in the public, commercial or not-for-profit sectors.

Competing interests None declared.

Patient consent Not required.

Ethics approval Research Ethics Committee, National Taiwan University Hospital.

Provenance and peer review Not commissioned; externally peer reviewed.

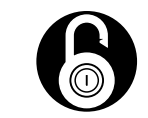

\section{OPEN ACCESS}

Open access This is an open access article distributed in accordance with the Creative Commons Attribution Non Commercial (CC BY-NC 4.0) license, which permits others to distribute, remix, adapt, build upon this work non-commercially, and license their derivative works on different terms, provided the original work is properly cited, appropriate credit is given, any changes made indicated, and the use is non-commercial. See: http:// creativecommons.org/licenses/by-nc/4.0/

(C) Author(s) (or their employer(s)) 2019. Re-use permitted under CC BY-NC. No commercial re-use. See rights and permissions. Published by BMJ.

- Additional material is published online only. To view please visit the journal online (http://dx.doi.org/ 10.1136/jnnp-2018-319268).

Check for updates 
To cite Chen C-H, Tang S-C, Cheng Y-W, et al. J Neurol Neurosurg Psychiatry 2019;90:841-843.

Received 19 July 2018

Accepted 17 September 2018

Published Online First 11 October 2018

J Neurol Neurosurg Psychiatry 2019;90:841-843.

doi:10.1136/jnnp-2018-319268

\section{REFERENCES}

1 Chabriat $H$, Joutel A, Dichgans $M$, et al. Cadasil. Lancet Neurol 2009:8:643-53.

2 Lee YC, Liu CS, Chang MH, et al. Population-specific spectrum of NOTCH3 mutations, MRI features and founder effect of CADASIL in Chinese. J Neurol 2009:256:249-55.

3 Liao YC, Hsiao CT, Fuh JL, et al. Characterization of CADASIL among the Han Chinese in Taiwan: distinct genotypic and phenotypic profiles. PLoS One 2015; 10:e0136501.

4 Lee JS, Ko K, Oh JH, et al. Cerebral microbleeds, hypertension, and intracerebral hemorrhage in cerebral autosomal-dominant arteriopathy with subcortical infarcts and leukoencephalopathy. Front Neurol 2017;8:203.

5 Lee JS, Kang CH, Park SQ, et al. Clinical significance of cerebral microbleeds locations in CADASIL with R544C NOTCH3 mutation. PLoS One 2015;10:e0118163. 